\title{
TAXONOMIA COMO ELEMENTO ESTRUTURANTE EM PORTAIS CORPORATIVOS
}

\author{
TAXONOMY AS A STRUCTURAL ELEMENT IN CORPORATE PORTALS
}

\author{
Luciane Paula Vital ${ }^{1}$
}

\section{RESUMO}

Este trabalho busca discutir os objetivos e requisitos necessários a um portal corporativo e de que forma a construção de uma taxonomia possibilita atingi-los, configurando-se em um modelo de representação da informação e do conhecimento nesse contexto. Apresenta a evolução das intranets em direção aos portais, construindo um ponto de acesso comum as informações organizacionais. Apresenta uma revisão sobre as funcionalidades que um portal corporativo deve apresentar. Conceitua e caracteriza os diferentes tipos de taxonomia e a forma com que organiza e representa a informação. Por fim, apresenta a taxonomia como ferramenta para a determinação de categorias conceituais e de relacionamentos semânticos de uma área, assim como, da estrutura navegacional, permitindo qualificar o processo de recuperação da informação.

\section{PALAVRAS-CHAVE}

Taxonomia. Portais corporativos. Representação da informação. Organização da informação

\begin{abstract}
This article discusses the objectives and requirements necessary for a corporate portal and how to build a taxonomy allows for to reach them, becoming a model of representation information and knowledge in this context. Shows the evolution of intranets into the portal, building a common access point to information organizational. Presents an overview of the features that an enterprise portal should present. Conceptualizes and describes the different types of taxonomy and the way it organizes and represents information. Finally, the taxonomy as a tool for determining the conceptual categories and semantic relationships of an area, as well as the navigational structure, allowing the process qualify for retrieval.
\end{abstract}

\section{KEYWORDS}

Taxonomy. Enterprise portal. Information representation. Information organization

\footnotetext{
1 Professora de Departamento de Ciência da Informação da Universidade Federal de Pernambuco. E-mail:
} lucianepv@yahoo.com.br.

(C) Rev. Digit. Bibl. Ciênc. Inf., Campinas, v.9, n.2, p.25-40, jan./jun. 2012 - ISSN 1678-765X 


\section{INTRODUÇÃO}

A gestão da informação e do conhecimento nas organizações não está somente atrelada a sistemas de informação com grande capacidade de processamento e infinitas possibilidades de cruzamento de dados, mas também a forma como as pessoas desenvolvem e compartilham conhecimentos. Para que esses processos gerem os resultados desejados é necessário se ater às informações inseridas nesses sistemas e, mais especificamente, nos processos de representação da informação, os termos utilizados para representar os conceitos contidos nos recursos de informação. A gestão da informação e do conhecimento encontra nos portais corporativos importante ferramenta para atingir seus objetivos, entretanto, o tratamento das informações e a codificação dos conhecimentos requerem o desenvolvimento e a aplicação de modelos de representação comuns a todos os usuários do sistema. A taxonomia, um modelo de representação de informação e conhecimento, vem sendo considerada uma metodologia facilitadora tanto da organização quanto da recuperação, representação e disponibilização do conteúdo em ambiente Web. Esse artigo busca discutir os objetivos e requisitos necessários a um portal corporativo e de que forma a construção de uma taxonomia possibilita atingir esses objetivos, configurando-se em um modelo de representação da informação e do conhecimento nesse contexto.

\section{PORTAIS CORPORATIVOS}

O surgimento dos portais na Internet está relacionado ao gerenciamento da crescente massa informacional criada e disponibilizada na Web. No final da década de 1990, os portais podiam ser caracterizados como sistemas de busca. Dias (2001, p.52) afirma que "para reduzir ainda mais o tempo de busca na Internet e auxiliar os usuários menos experientes, vários sites de busca incluíram categorias, isto é, passaram a filtrar sites e documentos em grupos préconfigurados de acordo com seu conteúdo [...]”. Essa forma de organizar e disponibilizar o acesso à informação tornou-se muito popular nos chamados 'portais públicos', que "[...] provêm ao consumidor uma única interface à imensa rede de servidores que compõem a Internet” (DIAS, 2001). Para Pérez de Leza (2000 apud ARCE, PÉREZ, 2001, p.223), um portal é definido como 
"uma página na Web que agrega conteúdos e funcionalidades, organizados de tal maneira que facilitam a navegação, proporcionam ao usuário um ponto de entrada na rede com um amplo leque de opções de serviços e produtos" ${ }^{2}$.

O objetivo inicial dos portais, fornecimento de um ponto de acesso comum a todo o conteúdo disponibilizado na Internet, $\log$ foi substituído por interesses primordialmente comerciais, cujo desejo é manter o usuário o maior tempo possível navegando no portal. Podemos citar como exemplos de portais públicos, o Yahoo (http://www.yahoo.com), o Bol (http://www.bol.uol.com.br), o Terra (http://www.terra.com.br) e tantos outros portais de provedores de acesso à Internet.

Os ambientes organizacionais logo se 'apropriaram' da funcionalidade do portal de ser um ponto de acesso único de informações e iniciaram, a partir das Intranets, a sua implantação. Nesse contexto, os portais podem ser concebidos como uma evolução das Intranets, pois integram vários sistemas de informação em uma plataforma única, com interface comum a todos os usuários. Com eles, a organização tem um 'ponto de encontro' quando se trata de recuperar ou disponibilizar informações, possibilitando, dessa maneira, potencializar o compartilhamento tanto de informações quanto de conhecimentos.

Podemos afirmar que portais representam uma evolução das Intranets quando entendemos Intranet como "[...] muito mais que uma mera interligação de servidores que executam tarefas semelhantes - é um sistema corporativo que envolve computadores e pessoas" (BENETT, 1997, p.21). Assim sendo, portais apresentam as principais características das Intranets, como a integração e o compartilhamento de informações, mas de modo centralizado. Carvalho e Ferreira (2005) discutem a evolução das Intranets em direção aos portais corporativos e declaram que "a missão dos portais corporativos é acabar com as ilhas dos sistemas de informações, integrando-as em uma única aplicação que seria a porta de entrada para todos os usuários do ecossistema empresarial". Portais utilizam metadados e linguagem XML (Extensible Markup Language), que possibilitam a integração de dados estruturados e não-estruturados. Dias (2001); Carvalho e Ferreira (2005) afirmam que não há um consenso na literatura em torno do

\footnotetext{
2 "La pagina Web que agrega contenidos y funcionalidades, organizados de tal manera que faciitan la navegación y proporcionan al usuario um punto de entrada em la Red con um amplio abanico de opciones". (PÉREZ DE LEZA, 2000 apud ARCE, PÉREZ, 2001, p.223)

(c) Rev. Digit. Bibl. Ciênc. Inf., Campinas, v.9, n.2, p.25-40, jan./jun. 2012 - ISSN 1678-765X 
conceito de portais, são utilizados como sinônimos de Portais de Informações Empresariais, Portais de Negócios e Portais Corporativos.

Porém, apesar das ambigüidades, a definição de Portal de Informação Empresarial (EIP - Enterprise Information Portal) encontrada na literatura dá ênfase maior ao gerenciamento informacional, sem abordar de forma satisfatória o aspecto cooperativo (DIAS, 2001); e, quando trata desse aspecto, envolve o conceito de cooperação em um processo mais amplo de gestão do conhecimento. As tecnologias de EIP trabalham com os conceitos tratados anteriormente, de integração e compartilhamento de informações, tanto internas quanto externas ao ambiente organizacional. De acordo com Turban, Rainer Junior e Potter (2003, p. 233), os EIP “[...] integram aplicações de gerenciamento de conteúdo, inteligência empresarial, depósito de dados, central de dados e gerenciamento de dados". Centralizando o ponto de acesso às informações organizacionais, internas e externas, a empresa ganha em inteligência, melhora o compartilhamento e a produção de novos conhecimentos, além de qualificar as tomadas de decisões.

O termo Portal de Negócios, utilizado por Eckerson (apud DIAS, 2001), é entendido como "um aplicativo capaz de proporcionar aos usuários um único ponto de acesso a qualquer informação necessária aos negócios, esteja ela dentro ou fora da corporação". Essa definição de Eckerson está relacionada com a gestão da informação, cujo ponto central é a disponibilização de informações referentes aos processos organizacionais, sem necessariamente gerir o conhecimento.

Já o Portal Corporativo, no conceito de Collins (2003 apud CARVALHO e FERREIRA, 2005), é “[...] uma interface personalizada de recursos on-line que permite que os

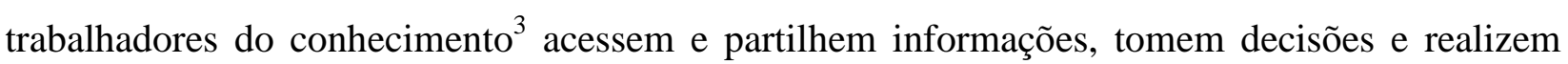
ações independentemente da sua localização física, do formato da informação e do local em que ela está armazenada". Terra e Gordon (2002) dizem que "os portais são fundamentais para organizações intensivas no uso de informação e conhecimentos, [assim como] para organizações que se comunicam e colaboram de forma intensiva com um número expressivo de agentes e

\footnotetext{
${ }^{3}$ Entende-se por trabalhador do conhecimento o indivíduo "[...]que sabe selecionar, absorver informação e conhecimento onde quer que ele se encontre e com capacidade para aplicar este conhecimento em ações concretas". (HARVARD BUSINESS REVIEW, 2000)
} 
atores externos (clientes, parceiros, fornecedores)". Segundo os autores, os portais melhoram a eficiência do trabalho em equipe, o uso de informações corporativas e do ambiente externo e podem aumentar a produtividade do 'trabalhador do conhecimento'. Ele aborda os portais corporativos dentro de um processo de Gestão do Conhecimento e os denomina 'Portais de Conhecimento Corporativo' (PdCC).

Eckerson (1999 apud DIAS, 2001) verifica uma evolução dos portais corporativos e os divide em quatro gerações (Quadro 1).

QUADRO 1 - Gerações de portais corporativos

\begin{tabular}{|c|c|c|}
\hline Geração & Categoria & Características \\
\hline Primeira & Referencial & $\begin{array}{l}\text { Máquina de busca, com catálogo } \\
\text { hierárquico de conteúdo da Web. } \\
\text { Cada entrada do catálogo contém } \\
\text { uma descrição do conteúdo e um } \\
\text { link. Essa geração enfatiza mais a } \\
\text { gerência de conteúdo, } \\
\text { disseminação em massa das } \\
\text { informações corporativas e o } \\
\text { suporte à decisão. }\end{array}$ \\
\hline Segunda & Personalizado & $\begin{array}{l}\text { O usuário, por meio de um } \\
\text { identificador e uma senha, pode } \\
\text { criar uma visão personalizada do } \\
\text { conteúdo do portal, conhecida } \\
\text { como "Minha Página". Essa visão } \\
\text { mostra apenas as categorias que } \\
\text { interessam a cada usuário. O } \\
\text { portal pode avisar ao usuário } \\
\text { sempre que um novo conteúdo for } \\
\text { adicionado às categorias por ele } \\
\text { assinaladas. Os usuários podem } \\
\text { publicar documentos no } \\
\text { repositório corporativo para que } \\
\text { esses sejam também visualizados } \\
\text { por outros usuários. Essa geração } \\
\text { privilegia a distribuição } \\
\text { personalizada do conteúdo. }\end{array}$ \\
\hline Terceira & Interativo & $\begin{array}{l}\text { O portal incorpora aplicativos que } \\
\text { melhoram a produtividade das } \\
\text { pessoas e equipes, tais como } \\
\text { correio eletrônico, calendários, } \\
\text { agendas, fluxos de atividades, } \\
\text { gerência de projeto, relatórios de } \\
\text { despesas, viagens, indicadores de }\end{array}$ \\
\hline
\end{tabular}




\begin{tabular}{|l|l|l|}
\hline & & $\begin{array}{l}\text { produtividade etc. Essa geração } \\
\text { adiciona oaráter cooperativo ao } \\
\text { portal, provendo múltiplos tipos } \\
\text { de serviços interativos. }\end{array}$ \\
\hline Quarta & Especializado & $\begin{array}{l}\text { Portais baseados em funções } \\
\text { profissionais, para gerência de } \\
\text { atividades específicas na na } \\
\text { instituição, tais como vendas, } \\
\text { finanças, recursos humanos, etc. } \\
\text { Essa geração envolve a integração } \\
\text { de aplicativos corporativos com o } \\
\text { portal, de forma que os usuários } \\
\text { possam executar transações, ler, } \\
\text { gravar e atualizar os dados } \\
\text { corporativos, ainda incorpora } \\
\text { outras possibilidades como } \\
\text { comércio eletrônico, por exemplo. }\end{array}$ \\
\hline
\end{tabular}

FONTE - ECKERSON, 1999 apud DIAS, 2001.

O portal corporativo evoluiu no sentido de disponibilizar conteúdos específicos para usuários definidos, procurando abrir um canal de compartilhamento entre os colaboradores e destes com os clientes da organização.

Atualmente, no cenário competitivo em que todas as organizações se encontram, arriscaríamos afirmar que a implantação de portais é fundamental em todas as organizações, pois informação e conhecimento são elementos essenciais para a gestão dos negócios. Cardoso (2002) aponta as seguintes vantagens dos portais:

- permitem aos departamentos de Tecnologia da Informação aumentar os benefícios dos datawarehouses ${ }^{4}$, data marts ${ }^{5}$ e outros projetos de Business Intelligence ${ }^{6}$;

- aumentam a eficiência da organização: os utilizadores pouparão tempo na busca da informação;

- aumentam a produtividade: melhor informação leva diretamente a melhores decisões. Adicionalmente, a possibilidade de tomar decisões rápidas significa freqüentemente diferença entre ganhar/perder um negócio;

4 "Data wharehouse é um grande banco de dados que armazena dados de diversas fontes para futura geração de informações integradas, com base nos dados de funcionamento das funções empresariais operacionais de uma organização inteira" (REZENDE E ABREU, 2003, p.211).

5 Data mart se refere "a um depósito de dados que atende a certas áreas específicas da empresa e voltados para o processo decisório gerencial” (BARBIERI, 2001, p. 50).

${ }^{6}$ Constitui-se na utilização de variadas fontes de informação para se definir estratégias de competitividade nos negócios das empresas.(BARBIERI, 2001)

(C Rev. Digit. Bibl. Ciênc. Inf., Campinas, v.9, n.2, p.25-40, jan./jun. 2012 - ISSN 1678-765X 
- redução nos custos: o portal self-service reduz os custos, evitando que os administradores passem horas a produzir relatórios e documentos;

- e-commerce ${ }^{7}$ : melhoramento nas relações com fornecedores, distribuidores e clientes;

- mais poderes para o utilizador final: o portal elevará a produtividade dos utilizadores, aumentando a sua independência.

Acrescentamos a essas vantagens o fato de possibilitar a integração, seja por meio de chats, fóruns, blogs, ou de qualquer outra ferramenta que permita o encontro e a troca de conhecimentos entre as pessoas. Dias (2003, p.69) declara que

[...] o grande desafio dos portais é o de ocultar os problemas relativos à heterogeneidade das fontes de informação e, assim, uma vasta produção científica tem se voltado para a integração de fontes heterogêneas de dados e a interoperabilidade de sistemas, com propostas que incluem a adoção de metadados, o uso de ontologias e outros recursos de integração [...]

Esses desafios encontram-se explicitados nas funcionalidades que os portais precisam apresentar, pois a heterogeneidade é inerente aos processos organizacionais. Sendo assim, devese encontrar uma forma de trabalhar com esses diferentes suportes e linguagens, com vistas a proporcionar o compartilhamento das informações.

Terra e Gordon (2002) dividem as funcionalidades em três grupos:

- Camada de Apresentação e Personalização define como os usuários enxergam e customizam a informação que é disponibilizada ou acessada pelo portal. As soluções mais intuitivas sendo oferecidas permitem aos usuários acessar a informação em ambientes mais relevantes e contextualizados.

- Taxonomias e Mecanismos de Busca determinam quão fácil será para os usuários encontrar informação relevante baseada em um conjunto de critérios de busca. [...] As soluções de busca têm-se desenvolvido para integrar variadas fontes de informação, tornando-se assim mais sofisticadas, intuitivas e adaptáveis às diferentes necessidades organizacionais.

- Aplicações Web. [...] Soluções sofisticadas de plataformas de portais fornecem uma série de recursos que tornam muito fácil integrar diferentes tipos de aplicações [...].(TERRA; GORDON, 2002, p. 95)

Essas funcionalidades, representadas na Figura 1, sucintamente objetivam que o usuário encontre informações pertinentes ao desenvolvimento do trabalho e tenha a possibilidade de colaborar por meio da codificação e disponibilização de seus conhecimentos.

${ }^{7}$ Comércio eletrônico.

(c) Rev. Digit. Bibl. Ciênc. Inf., Campinas, v.9, n.2, p.25-40, jan./jun. 2012 - ISSN 1678-765X 


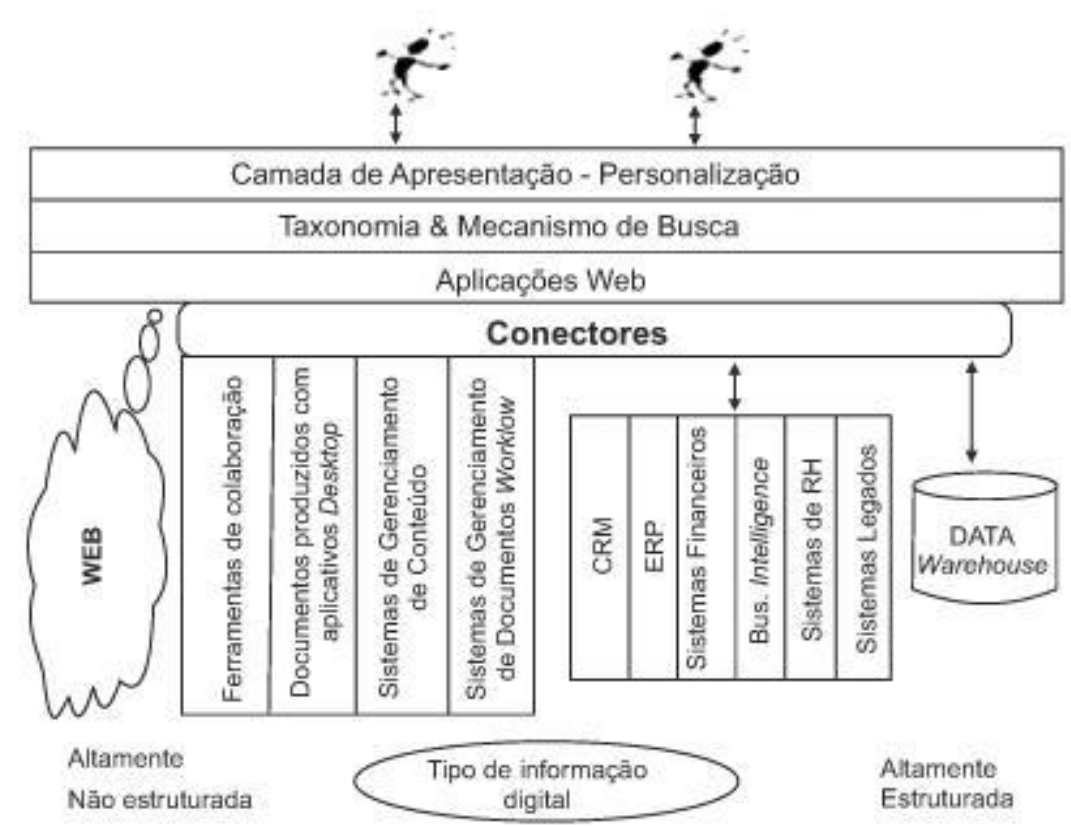

ARTIGO

FIGURA 1 - Componentes-chave da arquitetura PdCC

FONTE - TERRA ; GORDON, 2002, p. 96.

Os três grupos de funcionalidades - Camada de apresentação, Taxonomia e Mecanismo de busca e Aplicações Web - são suportados por conectores. Esses conectores podem ser sistemas de gerenciamento de conteúdo, de documentos, ferramentas de colaboração, softwares como, CRM (Customer Relationship Management) que trabalha com dados integrados de clientes, BI (Business Intelligence), que dá suporte para o gerenciamento informacional da organização, visando à tomada de decisão, entre outros.

Analisando os portais sob a ótica da Gestão do Conhecimento, Terra e Gordon (2002) ressaltam três aspectos essenciais:

- encontrar informações relevantes e fontes de informação;

- codificar e publicar seus conhecimentos;

- colaborar on-line.

Esses aspectos estão intimamente relacionados às três camadas de funcionalidades apresentadas na Figura 1. Por outro lado, as soluções de busca, que resultam em encontrar informações relevantes e fontes de informação, remetem-nos à camada de organização, categorização e criação de taxonomias nesse ambiente, principalmente quando se trata de buscar (C) Rev. Digit. Bibl. Ciênc. Inf., Campinas, v.9, n.2, p.25-40, jan./jun. 2012 - ISSN 1678-765X 
informações não-estruturadas, fundamentais em um processo de Gestão do Conhecimento, pois delas derivam os conhecimentos tácitos. Sendo assim, trataremos da forma como a taxonomia pode auxiliar na codificação, apresentação e recuperação de informações nesse contexto.

\section{TAXONOMIA}

A explosão informacional, decorrente dos avanços tecnológicos, da crescente robotização e automação das empresas, faz-nos antever profundas modificações nos ambientes corporativos. Diante desse quadro e motivada pela evolução tecnológica, em que os usuários adquirem um papel essencial tanto na produção como na categorização e uso das informações, surgiu a necessidade de meios automatizados de pesquisa e filtragem de informação, com o uso de taxonomias.

O termo taxonomia vem do grego taxis=ordem e onoma=nombre e derivou-se de um dos ramos da Biologia que trata da classificação lógica e científica dos seres vivos, fruto do trabalho do médico e botânico sueco Carolus Linnaeus (ou Karl von Linné). Porém, apesar de as taxonomias serem derivadas da Biologia, nos ambientes digitais seu aparecimento e uso, segundo autores como Edols (2001), Adams (2000) e Plosker (2005), está relacionado com as formas automatizadas de criação da informação, tornando-se alvo de estudos da Ciência da Informação.

No âmbito da Gestão do Conhecimento, as taxonomias são definidas como "[...] elementos estruturantes, estratégicos e centrais para negócios baseados em informação e conhecimento [...] para classificar e facilitar o acesso à informação" (TERRA, GORDON, 2002).

Para Martinez et al. (2004, p.106), "a taxonomia, em um sentido amplo, é a criação da estrutura (ordem) e dos rótulos (nomes) que ajudam a localizar a informação relevante. Em um sentido mais específico, é o ordenamento e rotulação de metadados, que permite organizar sistematicamente a informação primária". É interessante notar que a estrutura mais citada na literatura para a taxonomia é a hierárquica, sendo esta uma forma de caracterizá-la, como podemos perceber na Figura 2:

( Rev. Digit. Bibl. Ciênc. Inf., Campinas, v.9, n.2, p.25-40, jan./jun. 2012 - ISSN 1678-765X 


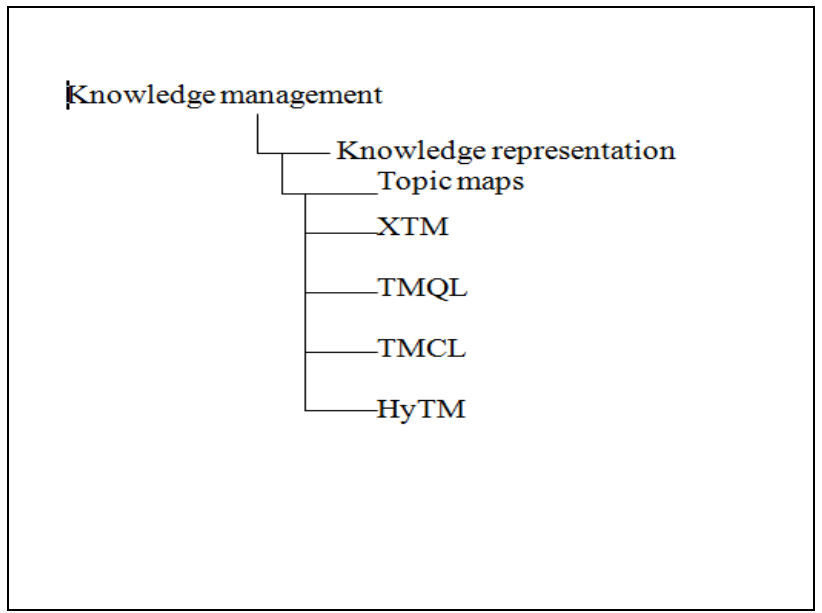

FIGURA 2 - Exemplo de taxonomia FONTE - GARSHOL, 2004.

A taxonomia organiza a informação da mais genérica à mais específica, utilizando-se da relação gênero-espécie entre os termos. Esse tipo de relação é definido por Dahlberg (1978) como sendo o que aparece "entre dois conceitos que têm idênticas características, sendo, porém, que de uma relação à outra é apresentada uma característica adicional, de modo que surge entre eles uma hierarquia". No entanto, as taxonomias corporativas não se limitam a essa relação somente, como veremos adiante.

O objetivo da taxonomia não é unicamente classificar e facilitar o acesso à informação, mas, igualmente,

[...] representar conceitos através de termos, agilizar a comunicação entre especialistas e outros públicos; encontrar o consenso; propor formas de controle da diversidade de significação e oferecer um mapa de área que servirá como guia em processo de conhecimento. (TERRA et al., 2005, p.1)

Adams (2000) explica que "[...] taxonomias organizam e criam significados para os relacionamentos entre coisas ou idéias"8. As organizações encontram dificuldade em prover acesso a informações semi-estruturadas (que podem estar nos servidores da empresa ou com grupos de usuários), e essa dificuldade é muito maior quando tratamos de informações que não apresentam estrutura alguma (armazenadas em arquivos pessoais, de acordo com a necessidade

8 "[...]Taxonomies order and give meaning to the relationship among things or ideas".(ADAMS, 2000, p.2)

(C) Rev. Digit. Bibl. Ciênc. Inf., Campinas, v.9, n.2, p.25-40, jan./jun. 2012 - ISSN 1678-765X 
dos indivíduos), mas que podem ser importantes para outras pessoas dentro da organização e precisam ser compartilhadas.

Taxonomias estão voltadas para a organização das informações em ambientes específicos, visando à recuperação eficaz. Para isso, estabelecem parâmetros em todo o ciclo de produção informacional, no qual profissionais distribuídos por espaços físicos distintos participam do processo de criação do conhecimento de forma organizada.

Como tratado anteriormente, o conceito de taxonomia, algumas vezes, é limitado a termos organizados em categorias hierárquicas; entretanto, quando tratamos de taxonomias em ambientes corporativos, já encontramos definições mais específicas.

Conway e Sligar (2002) classificam as estruturas taxonômicas, aplicadas em ambientes corporativos, em três tipos, de acordo com o objetivo a ser alcançado: taxonomia descritiva, de navegação e taxonomia para gerenciamento de dados.

A taxonomia descritiva consiste na criação de um vocabulário controlado com o objetivo de otimizar a busca e recuperação das informações. Baseia-se na estrutura de um tesauro, com o estabelecimento dos termos significativos em um determinado contexto, trabalhando com as relações de sinonímia, homonímia, etc. O objetivo é fazer com que essa taxonomia tenha significado no ambiente em que foi construída, buscando a padronização da linguagem a fim de que uma comunicação efetiva aconteça.

A taxonomia de navegação é baseada nos modelos mentais de organização das informações. A estruturação das informações fica visível ao usuário final, pois o objetivo é que a descoberta das informações aconteça na navegação. E, com base na observação desse comportamento de busca, a taxonomia vai modificando-se; conhecer o usuário nesse tipo de taxonomia é fundamental. As relações entre os termos precisam fazer sentido para os usuários dessa taxonomia, sem necessariamente apresentarem as subordinações lógicas.

A taxonomia para gerenciamento de dados tem como objetivo facilitar o compartilhamento em grupos específicos da organização, por exemplo, o setor de vendas. É composta de uma pequena lista de termos autorizados sem qualquer estrutura hierárquica, assemelha-se com as taxonomias descritivas; não tem, todavia, a pretensão de representar e promover acesso a toda informação produzida na organização.

(C) Rev. Digit. Bibl. Ciênc. Inf., Campinas, v.9, n.2, p.25-40, jan./jun. 2012 - ISSN 1678-765X 
Quanto à estruturação de uma taxonomia corporativa, Blackburn (2006) parte do pressuposto de que usualmente elas são hierárquicas e as divide em três tipos básicos: por assunto, por unidade de negócio e funcional.

A taxonomia por assunto, segundo a autora, faz uso de um vocabulário controlado. Organiza os termos por assuntos, partindo do mais geral aos mais específicos, por ordem alfabética, exigindo do usuário o conhecimento da área.

A taxonomia por unidade estabelece sua organização seguindo as unidades de negócio da organização. Apresenta como vantagem o fato de os indivíduos já estarem familiarizados com essa estrutura, não apresentando dificuldades em adotá-la. Entretanto, sempre que houver mudanças nessa estrutura, a taxonomia obrigatoriamente será modificada. Blackburn (2006) também mostra a dificuldade em trabalhar com documentos gerenciados ou compartilhados por várias unidades de negócios.

A taxonomia funcional é organizada levando-se em consideração as funções e atividades desenvolvidas pela organização. Os processos organizacionais mais gerais são utilizados para determinar também os níveis mais gerais da taxonomia. Já as funções formam os níveis intermediários, e as atividades o nível mais baixo, em uma estrutura hierárquica. Por exemplo, em uma agência reguladora do governo, Licenças e Certificações seria o processo geral; Inspeção, a Função; Notificação, a atividade. Apesar da divisão em três tipos distintos, Blackburn (2006) indica como mais apropriada a combinação das estruturas, levando em consideração o ambiente em que serão desenvolvidas.

Verifica-se que as classificações dos tipos de taxonomia propostas coincidem em alguns pontos. A taxonomia descritiva (CONWAY; SLIGAR, 2002) e a taxonomia por assunto (BLACKBURN, 2006) referem-se ao uso de um vocabulário controlado que estabelece a organização e controle de significação dos termos, objetivando melhorar a recuperação. Assim como, a taxonomia de gerenciamento de dados (CONWAY; SLIGAR, 2002) e a taxonomia funcional (BLACKBURN, 2006) visam representar a informação de setores específicos da organização. Podemos perceber que uma taxonomia corporativa, como forma de representar toda a informação disponível na organização (WOODS, 2004), não pode se restringir a apenas uma tipologia. A taxonomia corporativa precisa combinar os diferentes tipos a fim de desenvolver uma 
co-relação das linguagens funcionais usadas na organização (por meio da padronização dos termos e construção das relações semânticas); fornecer suporte para o mecanismo de busca (pela categorização dos resultados de busca); fornecer suporte e ferramentas de auxílio a um mecanismo de navegação (com base na estrutura taxonômica); além de conferir autoridade para qualquer usuário nomear e compartilhar documentos e outras informações no portal, buscando a explicitação do conhecimento (GILCHRIST, 2001).

Segundo Woods (2004), "uma taxonomia clássica supõe que cada elemento só pode pertencer a um ramo da árvore hierárquica. No entanto, em um ambiente corporativo isso não é possível nem desejável" (WOODS, 2004) ${ }^{9}$. Um documento pode ser de interesse de vários departamentos dentro da organização, com implicações e objetivos diferentes e precisa estar representado dentro desses diversos interesses, sem necessariamente se constituir apenas em uma relação gênero-espécie.

Outros tipos de relação entre conceitos, como as de funcionalidade também são importantes. A relação funcional é aquela em que, segundo Dahlberg (1978) "pode-se conhecer o caráter semântico [...] tendo por base as chamadas valências semânticas dos verbos [...]”. Valência semântica é caracterizada como "a soma dos lugares a serem preenchidos de acordo com a ligação deste conceito com outros" (DALHBERG, 1978). As relações entre os conceitos também podem ser compostas pelas relações intensionais, redes de conceitos, visto nas ontologias e essenciais nas taxonomias. A crescente produção informacional nas organizações, associada à disponibilização pela Internet de um volume cada vez maior de dados, faz com que a manutenção do controle sobre essa produção se torne uma tarefa praticamente impossível. Dessa maneira, a participação de representantes de todos os segmentos da organização no desenvolvimento da taxonomia permite ao usuário desempenhar o papel de criador, revisor, editor e publicador dos documentos que farão parte do portal corporativo.

Após o desenvolvimento de uma taxonomia, qualquer pessoa autorizada pode alocar as informações nos campos pré-determinados, tais como, newsletter, fale conosco, notícias, casos de sucesso, entre outros, fazendo com que a organização e a coerência no ambiente informacional sejam mantidas. A taxonomia, como qualquer outra forma de representação de informação e

9 "A classical taxonomy assumes that each element can only belong to one branch of the hierarquical tree. However, in a corporate environment, such formal ordering is neither feasible nor desirable". (WOODS, 2004)

(c) Rev. Digit. Bibl. Ciênc. Inf., Campinas, v.9, n.2, p.25-40, jan./jun. 2012 - ISSN 1678-765X 37 
conhecimento, precisa acompanhar a natureza dinâmica e as variações lingüísticas que ocorrem no fluxo informacional das organizações e, conseqüentemente, estar em constante manutenção.

\section{CONSIDERAÇÕES FINAIS}

Nas funcionalidades de um portal corporativo percebe-se a importância da categorização para organização e, consequente, recuperação das informações. Foi possível identificar a taxonomia como o modelo de representação que atende as necessidades do ambiente tanto do ambiente Web quanto do ambiente corporativo. As taxonomias buscam estabelecer categorias para a informação e apresentam os relacionamentos semânticos entre os termos, assim como, instituem uma estrutura para a navegação. Possibilitam ao usuário fazer parte do processo de construção, apresentando uma estrutura flexível, respondendo as necessidades de informação de ambientes específicos, com vistas à recuperação eficaz. Como citado anteriormente, faz com que profissionais distribuídos por espaços físicos distintos participem do processo de criação do conhecimento, através da representação e compartilhamento de informações, com o estabelecimento de categorias significativas. Originalmente as taxonomias são organizadas em estruturas hierárquicas, mas é perceptível, no ambiente corporativo, a necessidade do estabelecimento de relações associativas, que possam abranger, por exemplo, um elemento com significações diferentes dentro de cada uma das áreas da organização.

O processo de desenvolvimento de taxonomias está fundamentado nos conceitos de organização e representação da informação e do conhecimento e ainda requerem estudos teóricos que aprofundem seu desenvolvimento e prática, não só no ambiente corporativo, como nos demais contextos.

\section{REFERÊNCIAS}

ADAMS, K.C. Immersed in structure: the meaning a function of taxonomies. Internetworking, USA, n.3.2, ago. 2000. Disponível em:

http://www.internettg.org/newsletter/aug00/article_structure.html. Acesso em: 10 mar. 2010.

(C) Rev. Digit. Bibl. Ciênc. Inf., Campinas, v.9, n.2, p.25-40, jan./jun. 2012 - ISSN 1678-765X 
ARCE, M.V.S.; PÉREZ, T.S. Las comunidades virtuales y los portales como encenarios de gestión documental y difusión de información. Anales de Documentácion, n.4, p. 215-227, 2001. Disponível em: http://revistas.um.es/analesdoc/article/view/2311/2301. Acesso em: $02 \mathrm{fev}$. 2010

BARBIERI, C. BI - Business Intelligence: modelagem e tecnologia. Rio de Janeiro: Axcel, 2001.

BENETT, G. Intranets: como implantar com sucesso na sua empresa. Rio de Janeiro: Campus, 1997.

BLACKBURN, B. Taxonomy design types. AIIM E-doc Magazine, Maryland, USA. v.20, n.3, p.14-16, maio/jun. 2006.

CARDOSO, C.A. Porquê um portal organizacional. 2002. Disponível em:<http://kmol.online.pt/artigos/2002/01/01/porque-portal-organizacional>. Acesso em: 02 maio 2010.

CARVALHO, R.B.; FERREIRA, M.A.T. Análise das funcionalidades de intranets e portais: pesquisa exploratória em médias e grandes organizações brasileiras. In: ENCONTRO NACIONAL DE PESQUISA EM CIÊNCIA DA INFORMAÇÃO (ENANCIB), 6., 2005, Florianópolis. Anais... Florianópolis: PGCIN/UFSC, 2005. 1 CD-ROM.

CONWAY, Susan; SLIGAR, Char. Building taxonomies. In.: assets. Redmont: Microsoft Press, 2002. Cap. 6. . Unlocking knowledge

DAHLBERG, I . Teoria do conceito. Ci. Inf., Brasília, v. 7, n. 2, p.101-107, 1978.

DELPHI GROUP. Taxonomy and content classification. 2002. Disponível em: http://www.delphigroup.com/research.html. Acesso em: 15 mar. 2010.

DIAS, C.A. Portal corporativo: conceitos e características. Ci. Inf., Brasília, v. 30, n. 1, p.50-60, jan./abr. 2001.

. Usabilidade na Web: criando portais mais acessíveis. Rio de Janeiro: Alta Books, 2003.

EDOLS, L. Taxonomies are what? Free Pint, Reino Unido, n.97, out. 2001. Disponível em: < http://www.freepint.com/issues/041001.htm\#feature>. Acesso em: 10 nov. 2009.

GARSHOL, L.M. Metadata?Thesauri?Taxonomies?Topic Maps! Journal of Information Science, v.30, n.4, 2004. Disponível em: http://www.ontopia.net/topicmaps/materials/tm-vsthesauri.html. Acesso em: 10 fev. 2010 .. 
GILCHRIST, A. Corporate taxonomies: report on a survey of current practice. Online Information Review, v.25, n.2, p. 94-102, 2001.

HOLGATE, L. Creating and using taxonomies to enhance enterprise search. Information Today, Medford, N.J., v.7, n.21, jul./ago. 2004.

MARTINEZ, A.; et al. Las categorías o facetas fundamentales: uma metodología para el diseño de taxonomías corporativas de sitios Web argentinos. Ci. Inf., Brasília, v.33, n.2, p.106-111, maio/ago. 2004.

PLOSKER, G. Taxonomies: facts and opportunities for information professionals. Online. ABI/Inform Global, v.1, n.29, jan./fev. 2005. p. 58-60.

REZENDE, D. A.; ABREU, A.F. Tecnologia da informação aplicada a sistemas de informação empresariais: o papel estratégico da informação e dos sistemas de informação nas empresas. São Paulo: Atlas, 2003.

TERRA, J.C.C.; et al. Taxonomia: elemento fundamental para a gestão do conhecimento. 2005. Disponível em : http://www.terraforum.com.br. Acesso em: 12 nov. 2009.

; GORDON, C. Portais corporativos: a revolução na gestão do conhecimento. São

Paulo: Elsevier, 2002.

TURBAN, E.; RAINER JUNIOR, R.K.; POTTER, R.E. Administração de tecnologia da informação: teoria e prática. Rio de Janeiro: Elsevier, 2003.

WOODS, E. The corporate taxonomy: creating a new order. KMWorld, USA, v.13, n.7, jul., 2004. Disponível em: http://www.kmworld.com/Articles/ReadArticle.aspx?ArticleID=9566. Acesso em: 15 mar. 2010.

YAMAOKA, E.J. Taxonomia e metadados como elementos estruturantes da organização e representação do conhecimento numa empresa. 2005. 151f. Dissertação (Mestrado em Ciência da Informação) - Universidade de Brasília, Brasília, 2005. 